\title{
Towards Real-time Process Monitoring and Machine Learning for Manufacturing Composite Structures
}

\author{
Simon Stieber*, Alwin Hoffmann*, Alexander Schiendorfer*, Wolfgang Reif*, \\ Matthias Beyrle ${ }^{\dagger}$, Jan Faber ${ }^{\dagger}$, Michaela Richter ${ }^{\ddagger}$ and Markus Sause ${ }^{\ddagger}$ \\ ${ }^{*}$ Institute for Software \& Systems Engineering, University of Augsburg, Augsburg, Germany \\ Email: \{stieber, hoffmann, schiendorfer, reif\}@isse.de \\ ${ }^{\dagger}$ DLR Center for Lightweight Production Technology, German Aerospace Center (DLR), Augsburg, Germany \\ Email: \{matthias.beyrle, jan.faber\}@dlr.de \\ $\ddagger$ Institute of Materials Resource Management, University of Augsburg, Augsburg, Germany \\ Email: $\{$ michaela.richter, markus.sause $\}$ emrm.uni-augsburg.de
}

\begin{abstract}
Components made from carbon fiber reinforced plastics (CFRP) offer attractive stability properties for the automotive or aerospace industry despite their light weight. To automate the CFRP production, resin transfer molding (RTM) based on thermoset plastics is commonly applied. However, this manufacturing process has its shortcomings in quality and costs. The project CosiMo aims for a highly automated and costattractive manufacturing process using cheaper thermoplastic materials. In a thermoplastic RTM (T-RTM) process, the polymerization of $\epsilon$-caprolactam to polyamide 6 is investigated using an "intelligent tooling". Multiple sensor types integrated into the mold allow for tracking of several process-relevant variables, such as material flow and state of polymerization. In addition to the evaluation of the T-RTM process, a digital twin helps to visualize progress and to make predictions about possible problems and countermeasures based on machine learning. In this paper, the combination of software and hardware developments is described which will help to validate an optimal process setup for an industrial CFRP demonstrator.
\end{abstract}

\section{INTRODUCTION}

The use of light-weight components in automotive or aerospace vehicles is a key factor to reduce emissions and energy consumption [1]. Composite structures made of carbon fiber reinforced plastics (CFRP) make vehicles lighter by $50 \%$ to $70 \%$ compared to conventional metal structures and, thus, are more energy-efficient. Concerning automation, resin transfer molding (RTM) is the choice for manufacturing CFRP in a highly productive and cost-efficient manner. In the first step, a dry fiber preform is inserted into a two-piece mold tooling. After closing the tooling, liquid reactive resin is injected under high pressure until the fibers are thoroughly wetted. Assisted by the heated tooling, a curing process takes place after full impregnation. In the last step, the tooling is opened and the finished composite product can be removed.

Although originally established for thermosets, the RTM process has been adapted for thermoplastic materials like polyamide 6 (PA6), which is cheaper and allows for recycling,

This paper presents results of the research project MAI CC4 CosiMo (Composites for sustainable Mobility). The authors would like to thank the Bavarian State Ministry of Economic Affairs and Media, Energy and Technology (StMWi) for its financial support within the Campus Carbon 4.0 program. Further thanks go to the Project Management Jülich (PtJ) and MAI Carbon for their support. welding, and additional forming capacities (then called $\mathrm{T}$ RTM). In this case, liquid $\epsilon$-Caproclactam melt is used instead of the reactive resin and the curing process is replaced by an in-situ polymerization into PA6. Using the starting monomer Caprolactam with a low viscosity instead of remelting the long-chain polymer PA6 for the infiltration process allows for an excellent impregnation of the preform.

However, producing ready-made components in a fully automatic manner is error-prone due to several challenges. For example, imperfect handling of the textile preform can lead to dry spots; fluctuations in the raw materials may cause inhomogeneous polymerization, and closed molds make it hard to diagnose or steer the process. To cope with such deficiencies, online monitoring via sensors installed in the mold tooling, followed by subsequent data analysis is a necessary step to adapt the process and to identify faulty products (cf. [2], [3]). In particular, the in-mold sensors can track the filling status at certain points throughout an injection - yielding a spatially and temporally distributed input field.

Having in-situ monitoring available opens up the possibility to feed the data to the edge analytics of a digital twin [4]. Detecting anomalies, predicting future flow front dynamics, determining the fiber volume contents of the textile, or predicting dry spots are among the application scenarios relevant to reducing T-RTM rejects. Machine learning (ML) methods are promising due to i) their adaptivity to slightly changed process settings via generalization and ii) the presence of measured sensor data which captures correlations beyond simple models.

\section{ObJectives AND System OVERVIEW}

In order to implement the overall system and its digital twin, we developed a system architecture in CosiMo which is depicted in Figure 1. It consists of three different parts: the T-RTM machinery, the in-situ sensors of the intelligent tooling, and finally the digital twin. This architecture and the digital twin, in particular, have been developed with the following objectives $\mathrm{O} 1$ - $\mathrm{O} 4$ in mind:

O1: Monitor the manufacturing process of every composite part with the in-situ sensors of the tooling. 


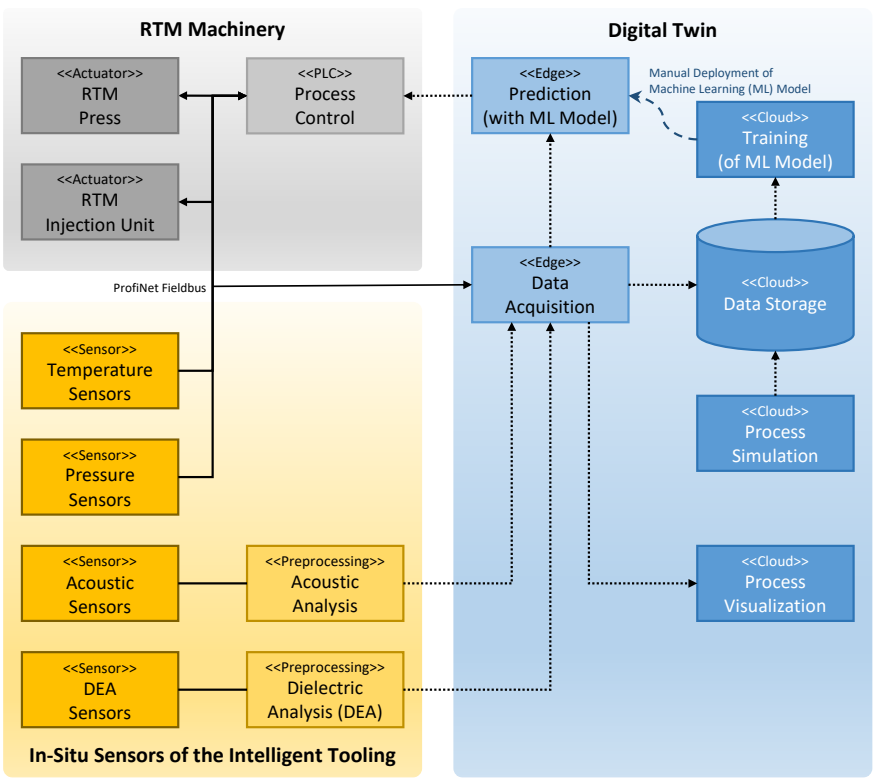

Fig. 1. Overview of the system architecture: Besides the RTM machinery, there are in-situ sensors inside the intelligent tooling. The digital twin records all data from the process and offers prediction models using machine learning.

O2: Visualize each manufacturing process in real-time to gain further process insights.

O3: Train ML models on simulated and real data for predicting the outcome of the manufacturing process.

O4: Use the trained ML models during manufacturing to reduce rejects.

The T-RTM machinery consists of two off-the-shelf components: a Wickert hot press with a pressing force of $4400 \mathrm{kN}$, and a T-RTM injection unit by KrausMaffei. Both are connected over the ProfiNet field-bus to a PLC which is responsible for controlling the overall manufacturing process. The pressure sensors as well as the temperature sensors of the tooling are also connected over the ProfiNet field-bus to the PLC. These two kinds of sensors are often used in classical RTM machinery to have minimal process feedback. However, to address objective O1, we use additional in-situ sensors, i.e., acoustic sensors as well as dielectric sensors, to achieve a better picture of the T-RTM process inside the intelligent tooling (cf. Section III). Both sensor types need real-time pre-processing to transform the raw data into higherlevel information about the flow front or the curing of the resin. The overall process setup is shown in Figure 2.

The preprocessed data of these two sensor types are recorded directly at the edge using a data acquisition component (i. e., ibaPDA from iba AG). The packets exchanged on the ProfiNet field-bus are recorded by the data acquisition component as well. Hence, all process and sensor data are recorded and merged with a consistent time stamp and it is possible to link the process data and part information with the sensor data (cf. objective O1). The ibaPDA is capable of forwarding the merged data in real-time to other components of the digital twin, in particular to cloud-based storage and

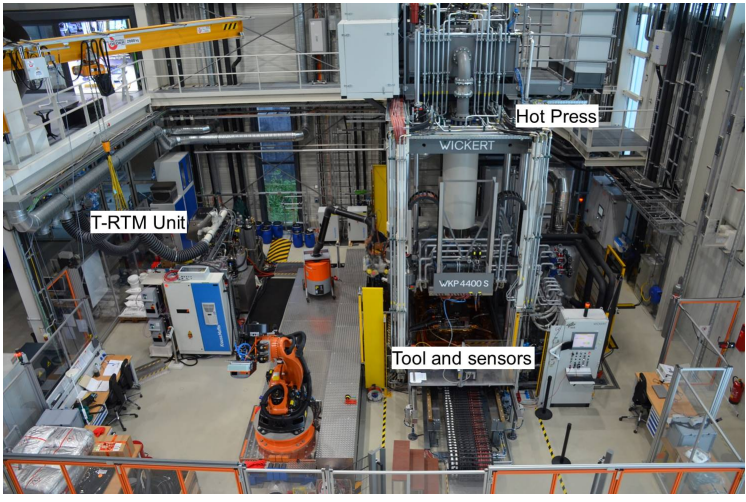

Fig. 2. Overview of the real process setup at DLR including a hot press, a T-RTM injection unit, and a sensor-integrated tooling.

visualization components as well as to a prediction component located at the edge (see Figure 1).

We plan to realize a component as part of the digital twin to visualize the manufacturing process based on the insitu sensors (cf. objective O2). This will enable composite engineers to better understand the dynamics inside the tooling during the T-RTM process and, thus, to identify problems as well as to manually optimize the parameters of the process. As all data is stored permanently in a data repository, it is also possible to replay and visualize selected manufacturing processes for analysis purposes.

To automatically optimize the manufacturing process, the stored data is used to train ML models to address objective O3. These models are responsible, e. g., for reconstructing the flow front of the resin from the sensor network, for predicting the flow front progress, and - ideally - for adapting the process parameters to optimize the result either during a process instance or for the subsequent runs. However, the amount of real data is generally not sufficient to train reliable models. That is why we make use of ESI PAM-COMPOSITES (PAMRTM module, Visual-RTM 15.5) to augment the measured data with simulated runs. Within this software, the real process is modeled for glass fiber nonwoven fabrics as reinforcement material. The challenge is to set up a simulation that describes reality as close as possible, knowing that the real process is subject to variations, e.g., a different fiber volume content (FVC). Due to these variations and pending validation of the kinematic models in the PAM-RTM software for the polymerization process of $\epsilon$-Caproclactam, a gap between simulation and reality is expected.

Because of the simulation-reality gap, we pursue a transfer learning approach (cf. Section IV). The (more abundantly available) simulated data is used to pre-train the model while the (scarce) real data is used to adapt the model to the real sensors and machinery. When a model is adapted to real data, it can be manually deployed as an edge analytics module (see Figure 1) to work with live data from ibaPDA. Hence, the model can make predictions to optimize the process manually or automatically by adapting the process parameters of the PLC (cf. objective O4). 


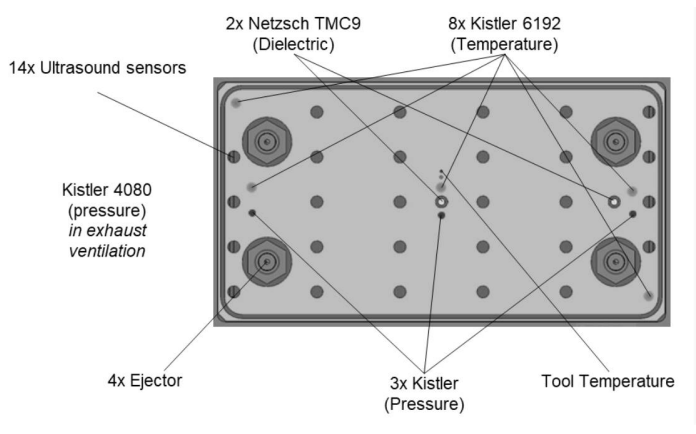

Fig. 3. Sensor types and arrangement at the plate tooling

\section{INTELLIGENT TOOLING}

In-situ sensor concepts are often mostly limited to temperature and pressure sensors. They can be beneficial for process control as they help to regulate the tooling temperature and the pumping pressure respectively. In the case of temperature sensors, studies were conducted regarding flow front detection [5] and curing monitoring [6]. However, they require close or direct contact with the cavity and a variety of material parameters. To improve the monitoring of the T-RTM process, we integrated different types of sensors into an intelligent tooling. Their continuous measurements enable insight into the filling of the tooling, the polymerization progress, and the detection of crack formation due to thermal and chemical shrinkage. The arrangement and type of sensors inside our tooling are shown in Figure 3.

Monitoring the polymerization progress can be achieved through dielectric analysis (DEA) where an alternating field is applied to the electrodes of the dielectric sensors. By measuring the electric permittivity, one can characterize the molecular mobility of ions and dipoles which is affected by the ongoing polymerization [7]. Several studies found correlations between DEA and differential scanning calorimetry (DSC) through kinetic observations [8], [7]. Moreover, the analysis of phase transitions for thermoplastic materials like carbon fiber reinforced PA6 shows the potential of DEA for characterizing the polymerization of $\epsilon$-Caprolactam to PA6 [9], [10].

In contrast to other methods, ultrasound measurement techniques require no direct contact with the resin and therefore allow monitoring without physical contact to the process. Using pulse-echo measurements, conclusions about the flow front position and polymerization progress can be drawn [11]. The arrival time of the flow front and the corresponding velocity at each sensor position is found by evaluating the amplitude of the echo caused by the transition between the tooling and the incoming resin. The polymerization progress can be tracked by measuring the sound propagation time in the resin layer, which is in reverse proportion to the velocity of sound of the material. It is also possible to detect structural damages caused by shrinkage and demolding as well as the occurrence of microscopic cracks by using acoustic emission analysis with the same ultrasound sensors. Moreover, continuous acoustic monitoring is suited for permanent monitoring of pumps,

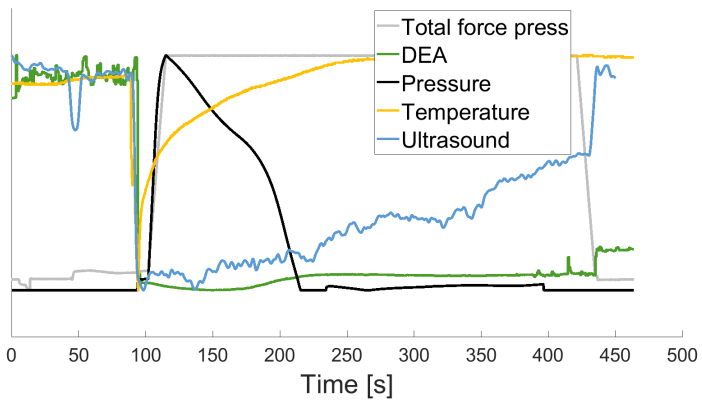

Fig. 4. Exemplary depiction of different sensor signals during the process.

valves, flow noise, and the filling process, which allows for the detection of abnormal states of the machinery in real-time.

Our research focus is on combining the signals from all sensor types to establish a comprehensive characterization of the process. Temperature, pressure, dielectric, and ultrasound sensors form a network of sensors that provide real-time measurements for discrete positions in the tooling. An example of measurement signals for different sensor types over time is depicted in Figure 4. The total force of the main press is included in light grey to show the closing of the tooling before the injection process. All sensor signals show significant changes during the process.

After performing the necessary data reduction steps, the results can be interpolated between the discrete positions to calculate the status inside the whole tooling. Hence, the polymerization progress and the position of flow front can be extended beyond the few known sensor positions. The discrete sensor positions have to be chosen carefully to find the right balance between enough interpolation points and the increasing cost with each additional sensor (see Figure 3). As ultrasound sensors provide the most information and can be manufactured with low cost, they form the backbone of a network with periodic distances and additional ones at complex geometry. DEA, pressure, and temperature sensors complement these with measurements at important positions.

\section{TRANSFER LEARNING}

In manufacturing in general and in composite manufacturing in particular, there are often not enough data for machine learning. Hence, our proposed remedy is to train models on simulated data which is easily available in high volume (cf. objective O3) After pre-training a model on simulated data, it is fine-tuned on real-world data. This strategy is commonly known as Transfer Learning and adopted successfully in computer vision [12] and other domains such as natural language processing [13].

To address the real-life issue of changes in the starting material - the textile, we used PAM-RTM to automatically by altering the FVC. More specifically, we added patches with lower and higher permeability, with values drawn from a random distribution, to get an irregular flow front and finally to provoke dry spots. By that, we hope to produce a database that is closer to reality due to noise and thus obtains more 
robust classifiers. Until now, the 2D simulation of a plate was simulated 40k times, resulting in $5 \mathrm{~m}$ frames. For geometries with higher complexity, this procedure can be repeated to collect data again. When compared to the amount of collected data in the real world, the advantages of simulated data become apparent. In the CosiMo project, the aim is to perform several hundreds of processes.

When using only simulation data to predict the future in the real world or to parameterize processes, the simulation-reality gap is often obstructive to the original cause. For the RTM process, in particular, there have been several studies on the gap between simulation and reality [14], [15], [16]. Factors such as capillary effects, multiple types of sensors with their accompanying noises, or timing issues make reality diverge from simulation. To bridge this gap for predictions in RTM, we devised the following steps. Since we do not only use pressure sensors in the real world (which is the only available sensor type in simulation), we extract data from the simulation results to mimic the data coming from the sensors described in Section III: We extract arrival and speed information of the flow front at certain points of the simulated tool.

A wider gap to cover will be the lack of fast adaptive changes in the viscosity. Caprolactam polymerizes very quickly at a certain temperature and therefore the advancement of the flow front has to be completed before polymerization. Sensing this crystalization is possible with the DEA sensors in our sensor network and has to be correlated with additional simulation data from PAM-RTM. To overcome all other differences between simulation and reality, we will fine-tune a model initially trained on simulated data using real data in a second step. By doing so, we hope to get a model that is more robust in reality than a pure simulation model. Furthermore, the fine-tuned model will perform much faster on a certain narrow task, such as classifying a dry spot, than the simulation model with wider abilities. This will enable on-line adaptations to the process, leading to a higher quality in components and a lower rejects rate (cf. objective O4).

A first learning approach only with simulation data was conducted [17] with differently sized networks of pressure sensors to classify if there are dry spots in the mold. By training a deconvolutional neural network to first learn a representation of the flow front and then to classify dry spots with a convolutional network, it outperformed feed-forward networks: for dry-spot classification, $91 \%$ accuracy on single frames was obtained with the densest sensor network.

\section{CONCLUSION}

In the project CosiMo, the RTM process is innovated at multiple levels. Instead of a regular thermoset resin, a thermoplastic resin is used which is more cost-effective, makes it possible to easily impregnate the textile, and resulting components can be melded and recycled. The sensor network consists of novel types of sensors that can monitor the process more thoroughly than state-of-the-art in-situ monitoring. The resulting data is used to create a digital twin that enables not just a visualization of the process, but also advanced analytics with the help of machine learning. The first steps towards this real-time process monitoring for RTM have been completed: the process, the sensor network, and data collection are set up, and the first successful injections have been carried out. From the machine learning perspective, an initial breakthrough on merely simulated data has been achieved. The next step is to bring the real world and simulation together.

\section{ACKNOWLEDGMENTS}

The authors would like to thank Werner Zumbrunnen-Plass and Tobias Seitz (iba AG), Frédéric Masseria (ESI GmbH), Dr. Alexander Chaloupka (NETZSCH-Gerätebau GmbH) and our partners from Krauss Maffei Technologies $\mathrm{GmbH}$ and Siebenwurst Formenbau $\mathrm{GmbH}$ for their support.

\section{REFERENCES}

[1] J. Heywood and D. MacKenzie, Eds., On the Road toward 2050: Potential for Substantial Reductions in Light-Duty Vehicle Energy Use and Greenhouse Gas Emissions. MIT, 2015.

[2] N. Pantelesis and E. Bistekos, "Process monitoring and control for the production of CFRP components," SAMPE Conference, pp. 5-9, 2012.

[3] C. Larrosa, K. Lonkar, and F. K. Chang, "In situ damage classification for composite laminates using Gaussian discriminant analysis," Structural Health Monitoring, vol. 13, no. 2, 2014.

[4] S. Stieber, "Transfer Learning for Optimization of Carbon Fiber Reinforced Polymer Production," Organic Computing: Doctoral Dissertation Colloquium 2018, 2018.

[5] G. Tuncol, M. Danisman, A. Kaynar, and E. M. Sozer, "Constraints on monitoring resin flow in the resin transfer molding ( $\mathrm{rtm}$ ) process by using thermocouple sensors," Composites Part A: Applied Science and Manufact., vol. 38, no. 5, 2007.

[6] K.-T. Hsiao, R. Little, O. Restrepo, and B. Minaie, "A study of direct cure kinetics characterization during liquid composite molding," Composites Part A: Applied Science and Manufact., vol. 37, no. 6, 2006.

[7] A. McIlhagger, D. Brown, and B. Hill, "The development of a dielectric system for the on-line cure monitoring of the resin transfer moulding process," Composites Part A: applied science and manufact., vol. 31, no. $12,2000$.

[8] M. Karcher, A. Chaloupka, F. Henning, S. Schmölzer, and E. Moukhina, "Determination of curing kinetics of a two-stage curing epoxy resin for high performance composites," J. Plastics Technology, vol. 2, 2016.

[9] A. Chaloupka, A. Wedel, I. Taha, N. Rudolph, and K. Drechsler, "Phase change detection in neat and fiber reinforced polyamide 6 using dielectric analysis," in Materials Science Forum, vol. 825, 2015.

[10] A. Wurm, R. Soliman, and C. Schick, "Early stages of polymer crystallization-a dielectric study," Polymer, vol. 44, no. 24, 2003.

[11] N. Liebers, M. Kleineberg, and M. Wiedemann, "Ultrasonic flow front, thickness and cure monitoring of liquid composite molding processes," 2017.

[12] H. C. Shin, H. R. Roth, M. Gao, L. Lu, Z. Xu, I. Nogues, J. Yao, D. Mollura, and R. M. Summers, "Deep Convolutional Neural Networks for Computer-Aided Detection: CNN Architectures, Dataset Characteristics and Transfer Learning," IEEE Trans. Med. Imaging, vol. 35, no. 5, 2016.

[13] J. Devlin, M.-W. Chang, K. Lee, and K. Toutanova, "BERT: Pre-training of Deep Bidirectional Transformers for Language Understanding," 2018. [Online]. Available: http://arxiv.org/abs/1810.04805

[14] H. Grössing, N. Stadlmajer, E. Fauster, M. Fleischmann, and R. Schledjewski, "Flow front advancement during composite processing: predictions from numerical filling simulation tools in comparison with realworld experiments," Polymer Composites, vol. 37, no. 9, 2016.

[15] M. Arnold, "Einfluss verschiedener Angussszenarien auf den Harzinjektionsprozess und dessen simulative Abbildung," Dissertation, TU Kaiserslautern, 2014.

[16] C. Hahn, "A Simulation Approach of Permeability Prediction for RTM Process Simulation," Dissertation, TU München, 2014.

[17] S. Stieber, N. Schröter, A. Schiendorfer, A. Hoffmann, and W. Reif, "FlowFrontNet: improving carbon composite manufacturing with CNNs," in Proc. Joint European Conference on Machine Learning and Knowledge Discovery in Databases (ECML-PKDD), 2020. 